\title{
Epidemiología de la neumonía del adulto adquirida en la comunidad
}

\author{
GONZALO VALDIVIA C.*
}

\section{EPIDEMIOLOGY OF COMMUNITY-ACQUIRED PNEUMONIA IN ADULTS}

Respiratory diseases represent the third cause of death in Chilean population, after cardiovascular diseases and malignancy. Fifty percent of deaths caused by respiratory diseases in adults are attributable to pneumonia. In Chile, they represent the main cause of death due to infectious diseases and the first specific one in senescent adults over 80 years old. The incidence and mortality of community acquired pneumonia (CAP) increase in both extreme ages of life (less than one year old and over 65 years old). In the population over 65 years old, mortality is extremely increased, rising to rates of 6.6 deaths per 1000 inhabitants. High variability in pneumonia hospitalization rate has been observed in different geographic areas, probably due to different medical criteria used to evaluate the severity of illness, access to healthcare systems and characteristics of the evaluated population. About $20 \%$ of patients affected with CAP require hospitalization due to the severity of pulmonary infection and to the risk of complications or death, and the necessity of healthcare resources are focused in these patients. Several clinical-epidemiological parameters able to modify clinical presentation and severity of pneumonia have been identified, such as advanced age, presence of co-morbidities, host immune competence, tobacco and alcohol consumption, place of acquiring the infection, etiology and environmental pollution.

Key words: pneumonia, epidemiology, morbidity, mortality.

\section{RESUMEN}

Las enfermedades respiratorias constituyen la tercera causa de muerte de la población chilena, siendo sólo superadas por las enfermedades del aparato circulatorio y los tumores malignos. El 50\% de los decesos por enfermedades respiratorias en el adulto son atribuibles a la neumonía, siendo en Chile la principal causa de muerte por enfermedades infecciosas y la primera causa específica de muerte en la población senescente mayor de 80 años. La incidencia y letalidad de la neumonía adquirida en la comunidad (NAC) se elevan en las edades extremas de la vida (bajo un año de edad y sobre 65 años). En la población sobre 65 años de edad, la mortalidad se eleva en forma alarmante, alcanzando a 6,6 muertes por 1.000 personas. Se ha observado una gran variabilidad en la tasa de hospitalizaciones por neumonía en diferentes áreas geográficas, probablemente determinada por diferencias en los criterios empleados por los médicos para evaluar la gravedad de los enfermos, accesibilidad a los sistemas de salud y las características de la población examinada. Se estima que cerca de $20 \%$ de los pacientes con NAC requieren ser manejados en el hospital debido a la gravedad de la infección pulmonar, concentrándose en esta población el mayor riesgo de complicaciones, muerte y demanda de recursos de salud. Se han identificado algunas variables clínico-epidemiológicas capaces de modificar la forma de presentación y la gravedad de la enfermedad, tales como la edad avanzada, presencia de co-morbilidades, estado inmune del huésped, consumo de tabaco y alcohol, lugar de adquisición de la infección, el microorganismo causal y la contaminación ambiental.

Palabras clave: neumonía, epidemiología, morbilidad, mortalidad.

\footnotetext{
* Departamento de Salud Pública, Facultad de Medicina. Pontificia Universidad Católica de Chile, Santiago, Chile.
} 


\section{MAGNITUD DEL PROBLEMA}

Desde hace 15 años, las enfermedades respiratorias constituyen una de las principales causas de muerte de la población chilena, siendo sólo superadas por las enfermedades del aparato circulatorio y los tumores malignos ${ }^{1}$ [II]. Durante el año 2000, las enfermedades respiratorias fueron responsables del $10,5 \%$ de las muertes comunicadas en el país, con un volumen de 8.287 defunciones. La mortalidad por enfermedades respiratorias en la población adulta fue 3,2 veces superior a la observada en la población infantil, con una tasa específica de 54,4 defunciones por cada 100.000 habitantes ${ }^{1}$. La categoría diagnóstica de neumonía concentra el $50 \%$ de las muertes por enfermedades respiratorias en el adulto, elevándose al $82 \%$ en la población pediátrica ${ }^{1}$.

Como causa específica de muerte, la neumonía (entidad diagnóstica que corresponde a las categorías comprendidas entre los códigos J12 y J18 de la Clasificación Internacional de Enfermedades, $\mathrm{X}$ revisión) ${ }^{2}$ constituyó la principal causa de muerte en el 5,4\% de los pacientes fallecidos en nuestro país (4.283 muertes por neumonía $)^{3}$. El 97\% de los casos fatales correspondió a neumonías en las que no hubo identificación del agente causal del cuadro respiratorio (J18: neumonía, organismo no especificado) ${ }^{1-3}$. La identificación del agente causal en la minoría de los casos, confirma las limitaciones señaladas en la literatura de los métodos de diagnóstico microbiológicos utilizados en la práctica clínica y debe contrastarse con las cifras reportadas en los estudios etiológicos que fluctúan entre 37 y $79 \%$. La ausencia de un examen diagnóstico único que abarque todo el espectro de potenciales patógenos respiratorios y la posibilidad de infección polimicrobiana que ocurre al menos en un $10 \%$ de los casos de neumonía, explican la baja frecuencia de identificación del agente causal.

La neumonía es la tercera causa específica de muerte en la población chilena, siendo la primera causa de deceso en los mayores de 80 años $^{1,3}$ [II]. La mortalidad en adultos entre $20 \mathrm{y}$ 64 años es relativamente baja (14,8 por 100.000 habitantes), siendo el riesgo de muerte 40 veces superior en los adultos mayores de 65 años (Figura 1).

La tendencia nacional de la mortalidad por neumonía indica que, pese a los importantes avances en el desarrollo de nuevas técnicas de diagnóstico, mayor acceso a la atención de salud y disponibilidad de antibióticos de alta eficiencia, esta patología infecciosa constituye un problema creciente y de difícil control, a pesar del mayor conocimiento de su historia natural [III]. En el 2001, la tasa de mortalidad específica por neumonía fue 28,4 muertes por 100.000 habitantes, cifra relativamente elevada comparada con otros países de América Latina ${ }^{1}$. La mortalidad por neumonía muestra una curva en forma de J, con mayores tasas de mortalidad en las edades extremas de la vida ${ }^{1,3}$ (Figura 1). En

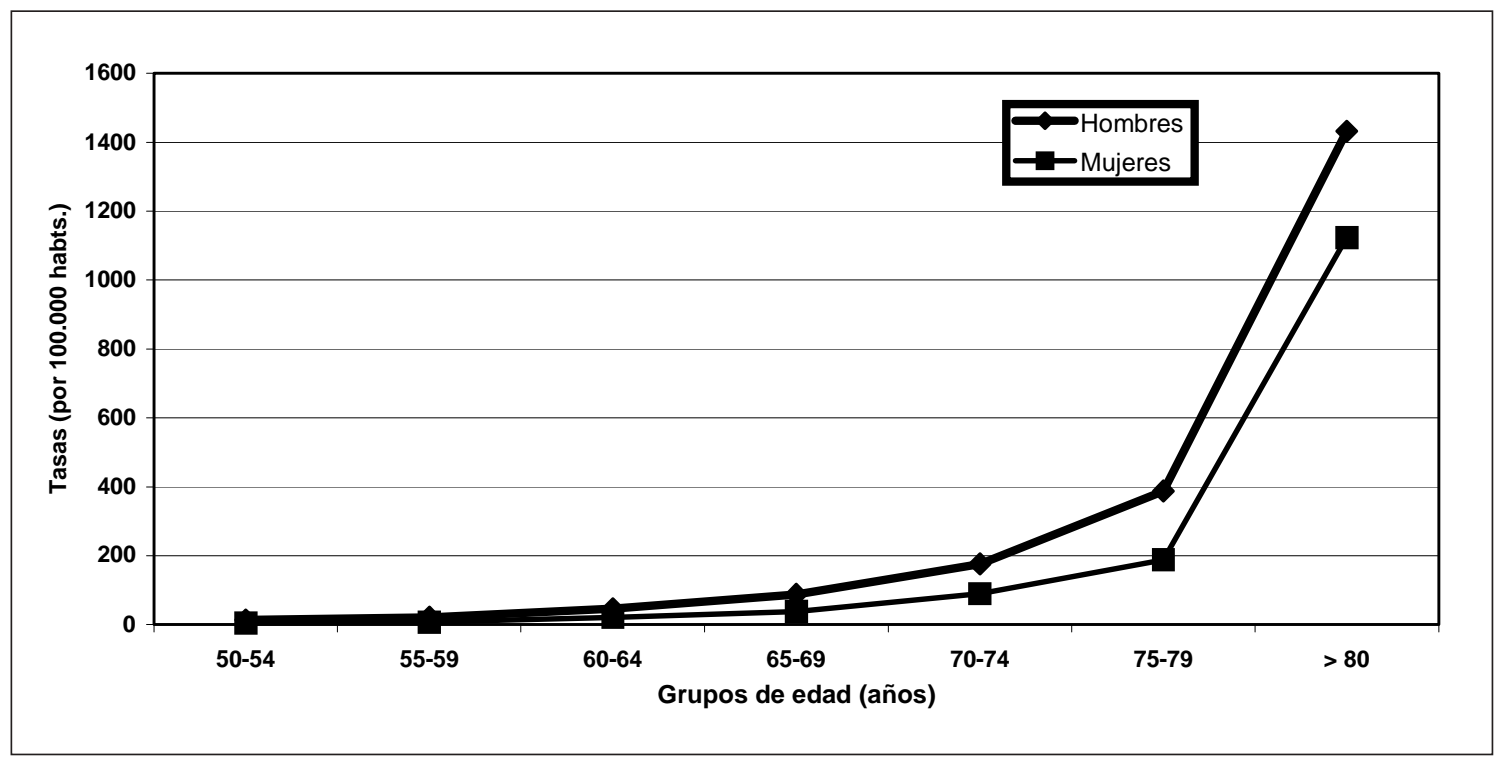

Figura 1. Tasas de letalidad por neumonía según sexo y grupo etáreo en Chile. Enero-Diciembre de 2000. 
la población mayor de 65 años, la mortalidad se eleva en forma alarmante, alcanzando a 6,6 muertes por 1.000 personas en esta categoría.

\section{TENDENCIA DE LA LETALIDAD POR NEUMONÍA}

En nuestro país, la mortalidad por neumonía ha declinado significativamente a partir de 1999 (46,4 muertes por 100.000 habitantes), probablemente debido a mejoría en las condiciones de acceso a los servicios de salud y oportunidad de tratamiento ${ }^{1,3}$. No obstante, la concentración de la mortalidad se ha desplazado al grupo de adultos mayores, contribuyendo a esta tendencia el paulatino envejecimiento de la población observado en los países desarrollados y en vías de desarrollo como el nuestro ${ }^{6}$ [II]. La incidencia y mortalidad de la neumonía tienen un patrón estacional en nuestro país, concentrándose en los meses de invierno. La distribución regional de la mortalidad por neumonía tiende a mostrar tasas más elevadas en los servicios de salud de la región austral del país ${ }^{6}$ (Figura 2).

\section{DEFINICIÓN OPERACIONAL}

La neumonía adquirida en la comunidad (NAC) se define como un cuadro infeccioso pulmonar, de carácter agudo, adquirido fuera de un establecimiento hospitalario, y que represen- ta un proceso incubado en el medio ambiente comunitario $^{4}$. La Sociedad Americana de Tórax (American Thoracic Society-ATS) no excluye de esta clasificación a los pacientes procedentes de ambientes institucionalizados, como casas de reposo o centros geriátricos, quienes presentan mayor riesgo de complicaciones y muerte ${ }^{7}$. Sin embargo, el diagnóstico de NAC puede presentar grados variables de heterogeneidad, lo que dificulta su análisis temporal y comparativo al utilizarse diferentes definiciones operacionales de la infección pulmonar ${ }^{8}$.

El diagnóstico clínico de NAC se sustenta en la presencia de dos o más síntomas de infección respiratoria aguda (fiebre, calofríos, tos, expectoración, dolor torácico, disnea), la constatación de infiltrados de aparición reciente en una radiografía de tórax, y la presencia de hallazgos semiológicos compatibles en el examen físico pulmonar (matidez, broncofonía, roncus, crepitaciones), en ausencia del antecedente de hospitalización durante al menos dos semanas previas al diagnóstico ${ }^{4,8}$. Usualmente se excluye de esta clasificación a los sujetos con algún grado de inmunodeficiencia; ya que el cuadro clínico, la etiología, evolución y pronóstico difieren significativamente en esta categoría.

Considerando que la NAC no es una condición sujeta a vigilancia epidemiológica, la estimación de su incidencia es incierta y se basa en información clínica que tiene grados variables de precisión, ya que se origina mayoritariamente de estudios centrados en pacientes hospitaliza-

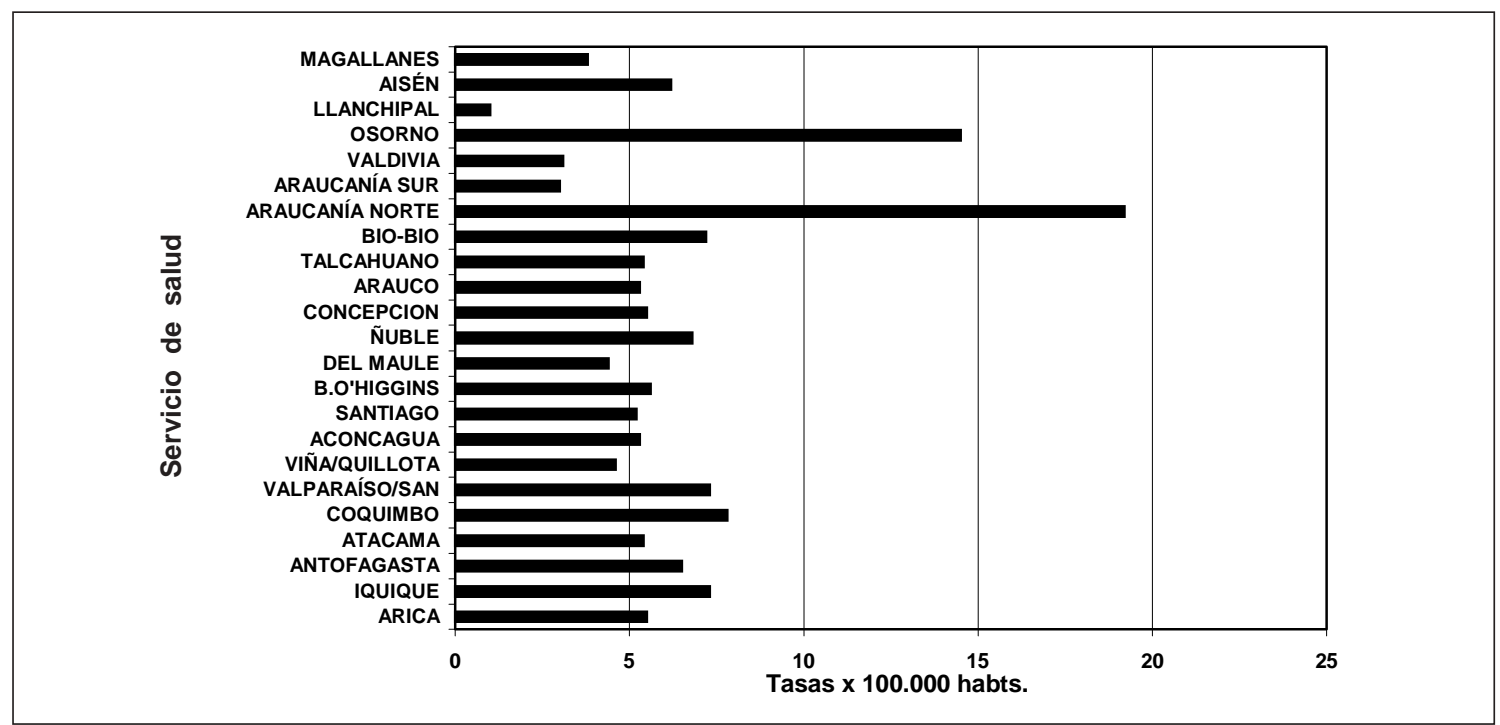

Figura 2. Tasas de mortalidad por neumonía según Servicio de Salud en Chile. Período 1999-2001. Fuente: Departamento de Estadísticas, Ministerio de Salud (Llanchipal = Llanquihue, Chiloé y Palena). 
dos. Estas estimaciones pueden no necesariamente reflejar las características epidemiológicas y el comportamiento de la enfermedad en la población general (en el contexto comunitario) por el conocido efecto de selección de los casos que consultan en estos centros asistenciales.

Sobre la base de las estadísticas regulares de salud, es complejo separar aquellos casos que corresponden estrictamente a NAC de aquellos originados en otros nichos epidemiológicos, especialmente intrahospitalarios ${ }^{1-3}$. La ausencia de datos regulares de morbilidad impide conocer con precisión el volumen de NAC de tratamiento ambulatorio. Según estimaciones de la ATS, cerca del $20 \%$ de las NAC pueden llegar a requerir hospitalización en algún momento de su evolución, ocurriendo generalmente en pacientes con características de riesgo particular ${ }^{4,8,9}$ [Ib, II, III]. La proporción de pacientes hospitalizados puede variar ampliamente y a veces depende de factores socioepidemiológicos no relacionados con el riesgo médico, comunicándose tasas de hospitalización que alcanzan el $64 \%$ de los casos diagnosticados sobre la base de estudios de carácter poblacional ${ }^{10}$. Se ha observado una gran variabilidad en la tasa de hospitalizaciones por neumonía en diferentes áreas geográficas, probablemente asociada a los diferentes criterios empleados por los médicos para evaluar la gravedad de los enfermos, accesibilidad a los sistemas de salud y las características de la población examinada ${ }^{4,8-10}$ [II] .
Considerando la existencia de programas específicos de control y manejo de las enfermedades respiratorias en Chile ${ }^{6}$, es posible que el porcentaje de hospitalización por NAC sea algo mayor, especialmente en pacientes menores de un año y adultos mayores de 65 años. En 2001 se registraron 78.661 egresos hospitalarios por neumonía, correspondiendo a $43,6 \%$ de todos los egresos por enfermedades respiratorias en los sistemas público y privado de salud ${ }^{6}$. Esto podría significar, de acuerdo a la estimación anterior, un volumen de más de 170.000 episodios de neumonía de manejo ambulatorio cada año en adultos mayores de 20 años (alrededor de 1 caso anual por cada 100 habitantes mayores de 20 años) $)^{4,6}$. La NAC corresponde a una entidad diagnóstica que compromete a todos los grupos de edad, estimándose las incidencias más elevadas en los menores de 15 años y en la población senescente (mayores de 65 años) $)^{6,11}$. La incidencia más alta se comunica en las personas mayores de 75 años ${ }^{11,12}$.

En nuestro país la distribución etárea de los pacientes hospitalizados por neumonía durante el año 2001, tanto en establecimientos públicos como privados, se ha concentrado fundamentalmente en la población menor de 4 años y en la población mayor de 65 años $^{6}$ (Figura 3) [II]. Cabe señalar que el $87 \%$ de los egresos por neumonía se originan en establecimientos del sistema público de salud, con estadías promedio similar en establecimientos públicos y privados

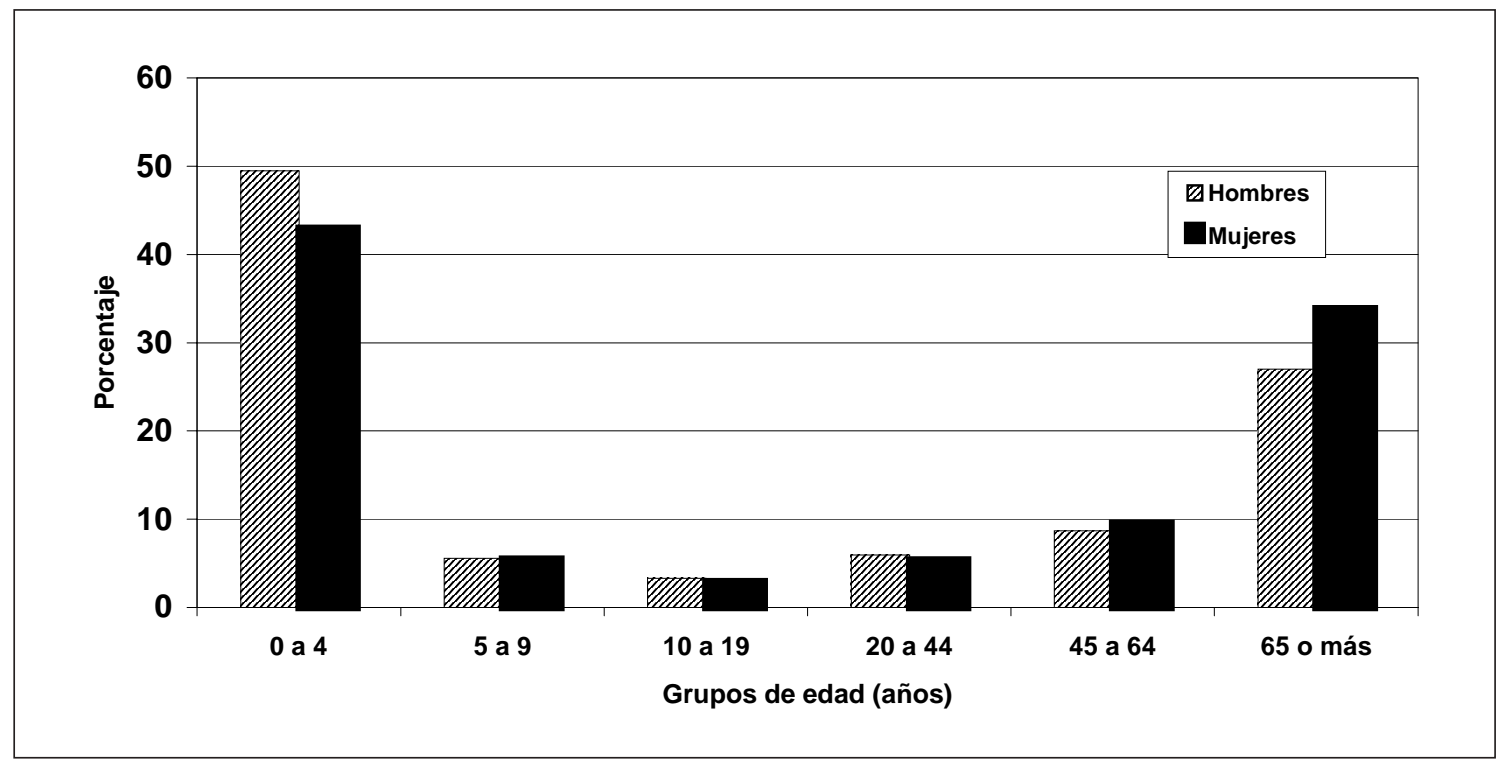

Figura 3. Porcentaje de egresos hospitalarios por neumonía según sexo y edad en relación al total de egresos por causas respiratorias. Enero-diciembre de 2001, Chile. 
(7,4 días por episodio de neumonía en promedio $)^{6}[\mathbf{I I}]$.

Durante ese mismo año, la letalidad de la neumonía fue de $5,7 \%$ en los establecimientos del sistema público y $3,7 \%$ en los establecimientos privados, debiendo considerarse que en el sector público se concentran los establecimientos de derivación nacional y regional, lo que origina un fenómeno de selección de los casos de mayor gravedad. La letalidad de la neumonía es mayor en la población senescente y particularmente entre los pacientes institucionalizados, lo que más probablemente refleja infecciones respiratorias propias del ambiente hospitalario, de conocido peor pronóstico vital comparado con las $\mathrm{NAC}^{4,8,9}$. La letalidad por neumonía en adultos hospitalizados en establecimientos de la Región Metropolitana en 1987 osciló entre 14 y 53\% (pacientes institucionalizados), probablemente reflejando distintas poblaciones y características de los centros asistenciales ${ }^{1,6}$ [III].

Universalmente se acepta que el riesgo de complicaciones y la letalidad de la NAC tratada en el medio ambulatorio (1-3\% de letalidad) es inferior a aquella que requiere ser manejada en el hospital (10-20\% de letalidad), especialmente en aquellos casos de neumonía grave que son manejados en las unidades de cuidado crítico (20-50\% de letalidad $)^{4,7-9,11-14}$ [IIb, II, III]

\section{LA NEUMONÍA ADQUIRIDA EN LA COMUNIDAD COMO PROBLEMA DE SALUD PÚBLICA}

Existen pocas condiciones médicas que tienen niveles de letalidad tan elevados como la $\mathrm{NAC}^{4,8,9}$. Por esta razón y por la elevada incidencia estimada, esta condición cumple con creces con los criterios epidemiológicos para ser considerada como un grave problema de salud pública. Implícitamente, el concepto de "comunitario" en materia de neumonías, puede interpretarse como de menor riesgo epidemiológico, lo que constituye un error. Se estima que la incidencia de las NAC es mayor en los países en desarrollo, lo que ha sido atribuido a una mayor prevalencia de tabaquismo en su población y deterioro en la situación medioambiental.

Los costos del cuidado de esta enfermedad han sido crecientes, concentrándose en los pacientes hospitalizados y admitidos a unidades de cuidado crítico por NAC grave ${ }^{4,15}$. Considerando que estamos en presencia de una patología infecciosa prevenible, cuya historia natural es conocida y que determina un quiebre en la calidad de vida especialmente en las edades extremas de la vida, la NAC tiene estatura suficiente para ser considerada como un problema de salud pública.

\section{FACTORES ASOCIADOS A LA PRESENTACIÓN DE LA ENFERMEDAD}

\section{Edad avanzada}

La NAC se presenta con mayor frecuencia en personas mayores de 65 años, constituyendo un factor de riesgo independiente que participa simultáneamente tanto en la incidencia como en la gravedad de la enfermedad ${ }^{4,8-10,16}$ [IIb, II]. Aunque esto carece de utilidad en términos prácticos, sí resulta relevante para efectos de focalizar recursos en los grupos más vulnerables de la población.

Las personas de edad avanzada presentan características anatómicas y funcionales muy particulares en su aparato respiratorio, con menor capacidad de respuesta a infecciones, mayor prevalencia de patologías crónicas (diabetes mellitus, cardiopatías, EPOC, insuficiencia renal crónica, neoplasias), presentando además un mayor riesgo de deficiencias nutricionales, lo que les confiere una especial labilidad biológi$\mathrm{ca}^{4,8,9,16}$. Enfermedades de alto catabolismo, como la neumonía, producen un grave deterioro del estado nutritivo que explica parcialmente la elevada letalidad en el anciano. En la próxima década, Chile incrementará significativamente su población de adultos mayores ${ }^{1}$. En dicho escenario poblacional, es improbable que la neumonía pierda su liderazgo como causa de muerte, tal como ocurre en países de elevado desarrollo industrial.

\section{Tabaquismo}

El hábito tabáquico constituye un factor de riesgo independiente de NAC y se cree que su alta prevalencia en países como el nuestro explica en parte la situación de la mortalidad por $\mathrm{NAC}^{1,3,6}$ [III]. Medidas epidemiológicas como el Riesgo Atribuible Poblacional Porcentual (RAPP), estiman que en sujetos portadores de enfermedad pulmonar obstructiva crónica, el tabaquismo podría explicar entre 23 y 32,4\% de los episodios de NAC en este grupo ${ }^{17}$.

Examinando el riesgo individual, se ha comunicado hasta 1,8 veces más riesgo de neumonía en fumadores habituales comparados con la población no fumadora, observándose además una tendencia positiva en relación a la duración del 
hábito ${ }^{7,17}$. La cesación del hábito tabáquico reduciría el riesgo de neumonía hasta en un 50\% después de 5 años. Por tanto, la fuerte asociación entre tabaquismo y neumonía, junto a la elevada prevalencia del hábito en la población, hacen recomendable insistir en una decidida intervención sobre este factor de riesgo. Los médicos y las autoridades de salud deben educar a la población sobre los riesgos específicos asociados al consumo de tabaco [C].

\section{Microorganismos involucrados en la NAC}

El perfil de agentes etiológicos involucrados en las NAC constituye una característica que podría diferenciarlas de las neumonías nosocomiales. Sin embargo, ha sido posible establecer que entre ambos tipos de neumonía puede haber sobreposición entre los agentes causales ${ }^{4,5,9,11,14}$.

Streptococcus pneumoniae es el principal agente etiológico asociado con la NAC (se estima que ocasiona por lo menos el $50 \%$ de los episodios del adulto), distribuyéndose universalmen$\mathrm{te}^{4,9,11}$ [IIb, III]. Al igual que en otros casos de microorganismos que conviven con el hombre colonizando sus epitelios, cambios en la ecología microbiana, en las características del huésped y en el medio externo e interno del sujeto resultan determinantes para la aparición de la enfermedad. En las últimas dos décadas, se ha comunicado un aumento significativo en la resistencia antibiótica de $S$. pneumoniae a penicilina, macrólidos y cefalosporinas, así como un aumento de la resistencia del Haemophilus influenzae a los agentes $\beta$-lactámicos ${ }^{4,18-20}$ [IIb, II]. Este fenómeno se explica por el amplio e indiscriminado uso de antibióticos en infecciones respiratorias virales en la comunidad y por la aplicación de esquemas terapéuticos no adheridos a protocolos consensuados para el tratamiento de enfermedades infecciosas pulmonares o sistémicas. En la medida que este fenómeno de resistencia progresa, se estima que la letalidad por NAC podría aumentar. Con el propósito de controlar la emergencia de patógenos respiratorios resistentes a los antimicrobianos en nuestro medio, sugerimos adherirse a las recomendaciones de las guías clínicas, evitar el uso de antibióticos en infecciones respiratorias agudas no complicadas (resfríos, faringitis, influenza, bronquitis) y apoyar la disposición del Ministerio de Salud que exige el uso de receta médica retenida para la prescripción de antimicrobianos a partir de $1999^{21}$ [C].

Los episodios epidémicos de infección por virus influenza en otoño-invierno constituyen un factor asociado temporalmente a mayor riesgo de NAC en nuestra población ${ }^{6}$ [II]. Los virus respiratorios asociados a fenómenos epidémicos pueden originar neumonías virales, a la vez que generan condiciones que facilitan la penetración de otros microorganismos a través de los epitelios dañados. El cambiante perfil de las cepas que originan los brotes de influenza obliga a mantener una conducta activa para detectar cambios en la incidencia y forma de presentación de las NAC. Las razones previamente señaladas justifican con plenitud la existencia de sistemas de vigilancia o de monitoreo microbiológico de las NAC a escala nacional, considerando su alta frecuencia poblacional y su elevada letalidad. La vacuna antigripal ha demostrado su eficacia en reducir la incidencia de influenza y el riesgo de NAC en la población de riesgo (mayores de 65 años y portadores de enfermedades crónicas) [Ia].

\section{Comorbilidad}

Se entiende por comorbilidad la existencia de patologías asociadas al cuadro clínico principal, lo que no significa que su importancia respecto al cuadro neumónico sea poco relevante. La presencia de comorbilidad en la población describe un elevado riesgo individual e inestabilidad respecto a la capacidad de respuesta a agresiones externas $^{4,9}$. Las mejores condiciones de vida de nuestra población permiten hoy que sujetos portadores de enfermedades crónicas tengan adecuadas expectativas de vida con escasas limitaciones $^{1,6}$. De acuerdo con la Primera Encuesta Nacional de Salud del año 2002, la prevalencia de enfermedades crónicas es elevada en nuestra población adulta ${ }^{22}$. La incidencia y gravedad de la NAC son mayores entre los pacientes con enfermedades crónicas, sean éstas respiratorias o de otro origen, y afectan tanto a pacientes pediátricos como adultos ${ }^{4,89}$ [Ia, Ib, II, III].

\section{Características medioambientales}

La progresiva importancia adquirida por las características medioambientales en el hogar ha permitido tener una visión más integral de esta variable en relación con las enfermedades respiratorias. Históricamente, se ha documentado una relación positiva entre la incidencia de NAC y los episodios agudos de contaminación del aire a $^{23,24}$ [II]. En la población infantil analizando la influencia de la contaminación intradomiciliaria, la evidencia indica aumento en el riesgo relativo de neumonía asociado al uso de combustibles fósiles en el hogar. En países desarrollados, con adecuado control de la contaminación ambiental, se han preocupado de controlar la contami- 
nación de origen intradomiciliario. En el ámbito mundial se estima que cerca del $50 \%$ de los hogares hacen uso de energía contaminante, lo que contrasta con el bajo porcentaje en países desarrollados del hemisferio norte. Si a esto se suma la exposición a tabaco en el ambiente, se configura un escenario epidemiológico de alto riesgo que expone a la población general a padecer infecciones respiratorias ${ }^{17,23,24}$. Esto, sin considerar que las características de la vivienda para un sector importante de la población generan condiciones de hacinamiento significativas, que probablemente puedan ser determinantes de la elevada incidencia de enfermedades respiratorias agudas encontrada en la población de bajo nivel socioeconómico.

\section{CONCLUSIONES}

La NAC del adulto constituye una importante fuente de morbimortalidad prevenible en el mundo $^{1-4,6}$. Las características de los agentes etiológicos involucrados en su desarrollo han sido mejor precisadas con el advenimiento de nuevas técnicas y procedimientos diagnósticos (incluyendo técnicas de biología molecular). A pesar de esto, y el desarrollo de medidas de soporte médico de avanzada, las NAC siguen exhibiendo una alta letalidad, causando muerte, en muchos casos prematura, especialmente en niños y ancianos, contribuyendo además a deteriorar significativamente la calidad de vida de los afec$\operatorname{tados}^{4,8,9}$.

Desde el punto de vista preventivo se vislumbra una interesante opción de intervención a través del desarrollo y aplicación de eficaces vacunas para algunos de los principales agentes etiológicos comprometidos en la NAC. Sin perjuicio de esto, debe insistirse en la necesidad de mejorar la calidad de la información disponible que permita conocer con mayor precisión la epidemiología y comportamiento de la NAC en nuestra población. En cuanto a los cuidados médicos otorgados a la población, la NAC ofrece la oportunidad de evaluar críticamente la gestión clínica, concordancia en el uso de arsenales terapéuticos atingentes con la realidad epidemiológica y la adecuada categorización de la gravedad del cuadro de neumonía utilizando los criterios disponibles para ello, ${ }^{4,8,9}$. El manejo de esta enfermedad infecciosa conlleva el gasto de importantes recursos económicos susceptibles de ser reducidos, considerando que se trata de una patología prevenible ${ }^{15}$. Esto implica la generación, difusión y adherencia a protocolos y guías clínicas consensuadas y la provisión de atención en cantidad y oportunidad adecuadas. Gran parte de los pacientes tratados por NAC serán manejados en sus hogares, lo que exige una conducta técnica clara y el adecuado apoyo de un equipo de salud convenientemente orientado.

\section{BIBLIOGRAFÍA}

1.- INSTITUTO NACIONAL DE ESTADÍSTICAS. Anuarios de Estadísticas Vitales, Chile, 2000.

2.- WORLD HEALTH ORGANIZATION. Statistical Classification of Diseases and Health Related Problems, 1993.

3.- SZOT J. Mortalidad por enfermedades respiratorias en Chile durante 1999. Rev Chil Enf Respir 2003; 19: 814.

4.- NIEDERMAN M S, MANDELL L A, ANZUETO A, BASS J B, BROUGHTON W A, CAMPBELL G D, et al. Guidelines for the management of adults with community-acquired pneumonia. Diagnosis, assessment of severity, antimicrobial therapy, and prevention. Am J Respir Crit Care Med 2001; 163: 1730-54.

5.- FERNÁNDEZ M, ZAGOLÍN M, RUIZ M, MARTÍNEZ M A, DÍAZ J C. Neumonía adquirida en la comunidad que se hospitaliza: estudio etiológico. Rev Méd Chile 2003; 131: 498-504.

6.- MINISTERIO DE SALUD DE CHILE. Departamento de estadísticas e información de salud (http:// deis.minsal.cl/index.asp).

7.- FARR B M, BARTLETT C L, WADSWORTH J, MILLER D L. Risk factors for community-acquired pneumonia diagnosed upon hospital admission. British Thoracic Society Pneumonia Study Group. Respir Med 2000; 94: 954-63.

8.- EWIG S. Community-acquired pneumonia: definition, epidemiology, and outcome. Semin Respir Infect 1999; 14: 94-102.

9.- FINE M J, SMITH M A, CARSON C A, MUTHA S S, SANKEY S S, WEISSFELD L A, KAPOOR W N. Prognosis and outcomes of patients with communityacquired pneumonia. A meta-analysis. JAMA 1996; 275: 134-41.

10.- ALMIRALL J, BOLIBAR I, VIDAL J, SAUCA G, COLL P, NIKLASSON B, et al. Epidemiology of community-acquired pneumonia in adults: a populationbased study. Eur Respir J 2000; 15: 757-63.

11.- WOODHEAD M A, MACFARLANE J T, Mc CRACKEN J S, ROSE D H, FINCH R G. Prospective study of the aetiology and outcome of pneumonia in the community. Lancet 1987; 1: 671-4.

12.- GOTFRIED M H. Epidemiology of clinically diagnosed community-acquired pneumonia in the primary care setting: results from the 1999-2000 respiratory surveillance program. Am J Med 2001; 111 (Suppl 9A): 25S-9S.

13.- MINOGUE M F, COLEY C M, FINE M J, MARRIE T J, KAPOOR W N, SINGER D E. Patients hospitalized after initial outpatient treatment for community-acquired pneumonia. Ann Emerg Med 1998; 31: 376-80.

14.- BRITISH SOCIETY RESEARCH COMMITTEE AND PUBLIC HEALTH LABORATORY SERVICE. The aetiology, management and outcome of severe community-acquired pneumonia on the intensive care unit. Respir Med 1992; 86: 7-13. 
15.- GUEST J F, MORRIS A. Community-acquired pneumonia: the annual cost to the National Health Service in the United Kingdom. Eur Respir J 1997; 10: $1530-4$

16.- SALDÍAS F, O`BRIEN A, GEDERLINI A, FARÍAS G, DÍAZ A. Neumonía adquirida en la comunidad en el anciano inmunocompetente que requiere hospitalización. Cuadro clínico, factores pronósticos y tratamiento. Arch Bronconeumol 2003; 39: 333-40.

17.- ALMIRALL J, GONZÁLEZ C A, BALANZO X, BOLIBAR I. Proportion of community-acquired pneumonia cases attributable to tobacco smoking. Chest 1999; 116: 375-9.

18.- DÍAZ A, TORRES C, FLORES L J, GARCÍA P, SALDÍAS F. Neumonía neumocócica adquirida en la comunidad en adultos hospitalizados. Rev Méd Chile 2003; 131: 505-14.

19.- PALAVECINO E. Puesta al día en el estudio de susceptibilidad de Streptococcus pneumoniae. Rev Chil Infect 2002; 19 (Supl. 2): S101-6.
20.- GONZÁLEZ P. Vigilancia de la resistencia a antimicrobianos. Rev Chil Infect 2002; 19 (Supl 2): S135-9.

21.- BAVESTRELLO L, CABELLO A, CASANOVA D. Impacto de medidas regulatorias en la tendencia de consumo comunitario de antibióticos en Chile. Rev Méd Chile 2002; 130: 1265-72.

22.- MINISTERIO DE SALUD DE CHILE. Departamento de Epidemiología. Encuesta Nacional de Salud. http:// epi.minsal.cl/epi/html/invest/ENS/ENS.htm.

23.- BURNETT R T, CAKMAK S, BROOK J R, KREWSKI D. The role of particulate size and chemistry in the association between summertime ambient air pollution and hospitalization for cardiorespiratory diseases. Environ Health Perspect 1997; 105: 614-20.

24.- SALINAS M, VEGA J. The effect of outdoor air pollution on mortality risk: an ecological study from Santiago, Chile. World Health Stat Q 1995; 48: 11825 . 